\title{
Frequent discordance in PD-1 and PD-L1 expression between primary breast tumors and their matched distant metastases
}

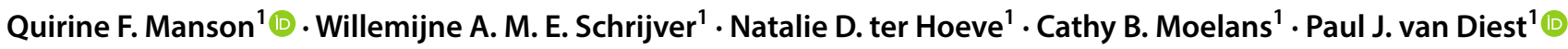

Received: 13 September 2018 / Accepted: 4 December 2018 / Published online: 13 December 2018

(c) The Author(s) 2018

\begin{abstract}
Programmed death-1 (PD-1) is an immune checkpoint that is able to inhibit the immune system by binding to its ligand programmed death-ligand 1 (PD-L1). In many cancer types, among which breast cancer, prognostic and/or predictive values have been suggested for both PD-1 and PD-L1. Previous research has demonstrated discrepancies in PD-L1 expression between primary breast tumors and distant metastases, however data so far have been scarce. We therefore evaluated immunohistochemical expression levels of PD-1 and PD-L1 in primary breast tumors and their paired distant metastases, and evaluated prognostic values. Tissue microarrays from formalin-fixed paraffin-embedded resection specimens of primary breast cancers and their matched distant metastases were immunohistochemically stained for PD-1 and PD-L1. PD-1 was available in both primary tumor and metastasis in 82 patients, and PD-L1 in 49 patients. PD-1 was discrepant between primary tumor and metastasis in half of the patients (50\%), PD-L1 on tumor cells was discrepant in 28.5\%, and PD-L1 on immune cells in $40.8 \%$ of the patients. In primary tumors there was a correlation between PD-1 positivity and a higher tumor grade, and between immune PD-L1 and ER negativity. In survival analyses, a significantly better overall survival was observed for patients with PD-L1 negative primary breast tumors that developed PD-L1 positive distant metastases (HR 3.013, CI 1.201-7.561, $\mathrm{p}=0.019$ ). To conclude, PD-1 and tumor and immune PD-L1 seem to be discordantly expressed between primary tumors and their matched distant metastases in about one-third to a half of the breast cancer patients. Further, gained expression of PD-L1 in metastases seems to indicate better survival. This illustrates the need of reassessing PD-1 and PD-L1 expression on biopsies of distant metastases to optimize the usefulness of these biomarkers.
\end{abstract}

Keywords Programmed death- $1 \cdot$ Programmed death-ligand $1 \cdot$ Breast cancer $\cdot$ Metastasis $\cdot$ Prognosis

Abbreviations

PD-1 Programmed death-1

PD-L1 Programmed death-ligand 1

TILs Tumor infiltrating lymphocytes

Paul J. van Diest

p.j.vandiest@umcutrecht.nl

Quirine F. Manson

q.f.manson@umcutrecht.nl

Willemijne A. M. E. Schrijver

w.a.m.e.schrijver@gmail.com

Natalie D. ter Hoeve

n.terhoeve@umcutrecht.nl

Cathy B. Moelans

c.b.moelans@umcutrecht.nl

1 Department of Pathology, University Medical Center Utrecht, PO Box 85500, 3508 GA Utrecht, The Netherlands

\section{Introduction}

Programmed death-1 (PD-1) is an immune checkpoint that is able to inhibit the immune system by binding to its ligand programmed death-ligand 1 (PD-L1), and prohibits T cell function in this way. In literature it has been described that in different cancer types [1], especially immunogenic tumors, including non-small cell lung cancer [2], malignant melanoma [3], and ovarian cancer [4], PD-1 can be upregulated on tumor infiltrating lymphocytes (TILs) and PD-L1 on tumor and immune cells. These markers could have potential prognostic and/or predictive values [5]. Although breast cancer is not considered very immunogenic, expression of PD-1 and PD-L1 has been described and prognostic and predictive values have been suggested, albeit with varying results [6-17].

Most studies focused on the expression of PD-1 and/or PD-L1 in primary breast tumors. However, through selective metastases of subclones, or tumor progression, expression 
levels and prognostic values in metastases may differ from the primary tumors. For example, previous research has described receptor conversion for ER, PR, HER2 and several molecular imaging targets in a significant proportion of breast cancer metastases [18, 19]. Additionally, it has been observed that conversion from ER/PR-positive primary breast cancers to ER/PR-negative metastases is associated with negative prognosis [20].

Since biomarker expression status of distant metastases, rather than the primary tumor will dictate response to targeted therapy, it is important to know whether conversion from the primary tumor to distant metastases also occurs for PD-1 and PD-L1. Two previous studies described high concordance of PD-L1 expression in primary breast tumors and their matched lymph node metastases, and lower PD-L1 expression levels in non-matched distant metastases, respectively [21, 22]. One study comparing PD-L1 expression between metastatic and non-metastatic primary breast cancers described one case where PD-L1 expression in primary tumor and metastasis was concordant, while in the second case expression was discordant [23]. As far as we know, these previous studies have only evaluated PD-L1 expression, while PD-1 expression may be of importance as well, certainly since brain metastases, compared to their primary breast tumor, are thought to have a lower amount of TILs [24].

Given the limited data on PD-1 expression and the anecdotal data on PD-L1 expression in primary breast cancers compared to their matched distant metastases to date, we compared immunohistochemical expression levels of PD-1 and PD-L1 in primary tumors and their matched distant metastases in a large group of metastatic breast cancer patients, and evaluated prognostic values.

\section{Materials and methods}

\section{Patients and samples}

For previous research, tissue microarrays (TMAs) with 106 primary female breast cancers and their matched distant metastases from various distant anatomical sites (bone, brain, gastrointestinal, gynecological (uterus/ovary), liver, lung/pleural and skin/subcutis) had been assembled [25, 26]. All metastases were metachronous metastases. Clinicopathological data (age, tumor size, histology, grade (according to the modified Bloom and Richardson score) [27], lymph node status, and surrogate molecular subtype, based on hormone receptor status- the ER/PR we used was, according to the Dutch guidelines with a cut-off of $10 \%$, and HER 2 was scored according to the ASCO/CAP guidelines) were anonymously extracted from digital reports/revised by a pathologist specialized in breast cancer (PvD). Follow-up data were anonymously obtained through the Comprehensive Cancer Center of The Netherlands (IKNL). Use of anonymous or coded left over material for scientific purposes is part of the standard treatment agreement with patients and therefore ethics approval and informed consent procedure was not required according to Dutch legislation [28].

\section{Immunohistochemistry}

The TMAs contained three cores of $0.6 \mathrm{~mm}$ from representative areas in formalin-fixed paraffin-embedded (FFPE) tissue blocks of both primary tumor and metastasis. $4 \mu \mathrm{m}$ thick tissue sections were cut and immunohistochemically stained with the Ventana autostainer (Roche, Tuscon, USA). For PD-1, we used a mouse anti-PD-1 monoclonal antibody (ab52587 (NAT105, dilution 1:50, Abcam, Cambridge, UK)) and for PD-L1, a rabbit anti-PD-L1 monoclonal antibody (741-4905 (clone sp263, dilution Ventana ready to use; Ventana Medical Systems, Tuscon, Arizona, USA)). ER (Ventana 1:100), PR (Dako 1:100) and HER2 (Neomarkers $1: 100)$ were also stained with the Ventana autostainer.

\section{Assessment of PD-1 and PD-L1}

The immunohistochemically stained slides were scored by consensus of two experienced observers (WS or QM and PvD), in random order and blinded to other data. PD-1 was scored positive in case of any membranous staining of immune cells. For PD-L1 we scored the positivity on both tumor cells and immune cells. Concerning PD-L1 on tumor cells, we scored the percentage of positive membranous staining. To create balanced groups for analyses, all cases with scores above 0 were considered to be positive. Immune PD-L1 was considered to be positive in case of any membranous staining of immune cells. Additionally, we verified the use of TMAs by comparing the PD-L1 scores of ten patients with PD-L1 scores on whole slides. In five cases, we observed complete agreement, and for the other five we only observed minimal differences.

\section{Statistics}

Statistical analyses were performed with IBM Statistics 25. To study differences in hormone receptor expression and expression of PD-1 and PD-L1 between primary tumors and (matched) metastases, McNemar and Pearson Chi square tests were used. To compare expression of PD-1 and PD-L1 in metastases and their anatomical sites, we used Fisher's exact test. For survival analysis, Kaplan-Meier curves were plotted and compared by log-rank test. Multivariate survival analysis was performed with Cox regression analyses. For all statistical tests, the significance level was set at $p$ value $<0.05$. 


\section{Results}

\section{Patient characteristics}

Of the 106 primary tumors, PD- 1 scores were obtained from 105 tumors and PD-L1 scores of 75 tumors. Regarding the 106 metastases, PD-1 scores were acquired of 83 tumors and PD-L1 scores of 67 tumors. Matched PD-1 scores were available for 82 tumors, while matched PD-L1 scores were available in 49 cases. Baseline clinicopathologic characteristics are shown in Table 1.

Mean age at surgery of the primary tumor was 53 years. The primary tumors had a mean size of $3.3 \mathrm{~cm}$, most were ductal $(88 \%)$, were grade 2 or 3 , and two-third of the samples was of the luminal subtype (66\%). More than half of the patients $(55 \%)$ had positive lymph nodes at diagnosis of the primary tumor.

Table 2 demonstrates the frequency of ER, PR, and HER2 positivity in primary tumors and metastases. No significant differences were observed between the primary tumors and metastases concerning ER and HER2, but PR was significantly less often expressed in metastases compared to primary tumors. Concordances between primary

Table 1 Basic clinicopathologic data for metastatic primary breast cancers

\begin{tabular}{ll}
\hline Characteristics & Primary breast tumor \\
\hline Age & \\
Mean [years (SD)] & $52.6(11.7)$ \\
Range & $27-85$ \\
Tumor size & \\
Mean [cm (SD)] & $3.3(2.6)$ \\
Range & $0.5-16.0$ \\
Histology ${ }^{\text {ano. }(\%)]}$ & \\
Ductal & $90(88.2)$ \\
Lobular & $12(11.8)$ \\
Grade [no. (\%)] & \\
1 & $3(3.0)$ \\
2 & $36(35.6)$ \\
3 & $62(61.4)$ \\
Lymph node status [no. (\%)] & \\
Negative & $45(44.6)$ \\
Positive & $56(55.4)$ \\
Molecular subtype [no. (\%)] & \\
Luminal & $69(65.7)$ \\
HER2 driven & $12(11.4)$ \\
Triple negative & $24(22.9)$ \\
\hline
\end{tabular}

${ }^{a}$ For the ductal type, also apocrine and metaplastic tumors were included
Table 2 Receptor status in primary breast tumors and non-matched distant metastases

\begin{tabular}{llll}
\hline Characteristics & Primary tumor & Metastasis & p-value* \\
\hline ER [no. (\%)] & & & \\
$\quad$ Negative & $44(40.0)$ & $39(47.0)$ & 0.332 \\
Positive & $66(60.0)$ & $44(53.0)$ & \\
PR [no. (\%)] & & & \\
$\quad$ Negative & $52(47.7)$ & $52(63.4)$ & $\mathbf{0 . 0 3 1}$ \\
$\quad$ Positive & $57(52.3)$ & $30(36.6)$ & \\
HER2 [no. (\%)] & & & \\
Negative & $88(82.2)$ & $66(79.5)$ & 0.635 \\
Positive & $19(17.8)$ & $17(20.5)$ & \\
\hline
\end{tabular}

Bold indicates $p<0.05$

*Pearson Chi square test

tumors and their matched metastases were for ER 94\%, for PR 78\%, and for HER2 95\% [Table 3].

\section{PD-1 and PD-L1 expression in primary tumors and matched distant metastases}

Figure 1 illustrates positive PD-1 and PD-L1 staining in primary tumors and matched distant metastases. The frequency of PD-1 immune cell expression, and PD-L1 tumor and immune cell expression in primary tumors and metastases is shown in Table 4, and did not differ between both groups.

The comparison between expression of PD-1 and PD-L1 between primary tumors and their matched distant metastases is shown in Table 5. For PD-1, 41/82 (50\%) primary tumors and metastases were concordant, while 17/82 (20.7\%) tumors converted from negative to positive and 24/82 (29.3\%) converted from positive to negative $(\mathrm{p}=0.349)$. For tumor PD-L1, $35 / 49(71.5 \%)$ primary tumors and metastases were concordant, while $3 / 49(6.1 \%)$ tumors converted from negative to positive and $11 / 49$ (22.4\%) converted from positive to negative $(\mathrm{p}=0.057)$. Concerning immune PD-L1, 29/49 (59.2\%) primary tumors and metastases were concordant, while 10/49 (20.4\%) tumors converted from negative to positive and 10/49 $(20.4 \%)$ converted from positive to negative $(\mathrm{p}=1.000)$.

As shown in Table 6, if PD-1 or PD-L1 (tumor or immune) was inconsistent in the primary breast tumor and metastasis, this was not always the case for the other protein. In only $47.9 \%$ of patients, both PD- 1 and tumor PD-L1 expression were comparable in the primary tumor and its metastasis, whereas this was the case for $60.9 \%$ of patients for both PD-1 and immune PD-L1 expression. 
Table 3 Receptor expression in primary breast tumors compared to their matched distant metastases

\begin{tabular}{|c|c|c|c|c|c|c|c|c|c|}
\hline \multicolumn{10}{|c|}{ Distant metastases } \\
\hline \multirow[t]{2}{*}{ Primary tumor } & \multicolumn{3}{|l|}{ ER } & \multicolumn{3}{|l|}{ PR } & \multicolumn{3}{|l|}{ HER2 } \\
\hline & Negative & Positive & p-value* & Negative & Positive & p-value* & Negative & Positive & p-value* \\
\hline Negative & $34(42.0 \%)$ & $1(1.2 \%)$ & 0.375 & $38(47.5 \%)$ & $5(6.3 \%)$ & 0.096 & $63(78.8 \%)$ & $3(3.8 \%)$ & 0.625 \\
\hline Positive & $4(4.9 \%)$ & $42(51.9 \%)$ & & $13(16.3 \%)$ & $24(30.0 \%)$ & & $1(1.3 \%)$ & $13(16.3 \%)$ & \\
\hline
\end{tabular}

*McNemar test

Fig. 1 Immunohistochemical positive staining for PD-1 (on immune cells) and PD-L1 (membranous staining on tumor and immune cells) in primary breast tumors and distant metastases. a PD-1 in a primary breast tumor. b PD-1 in a lung metastasis. $\mathbf{c}$ PD-L1 in a primary tumor. $\mathbf{d}$ PD-L1 in a lung metastasis
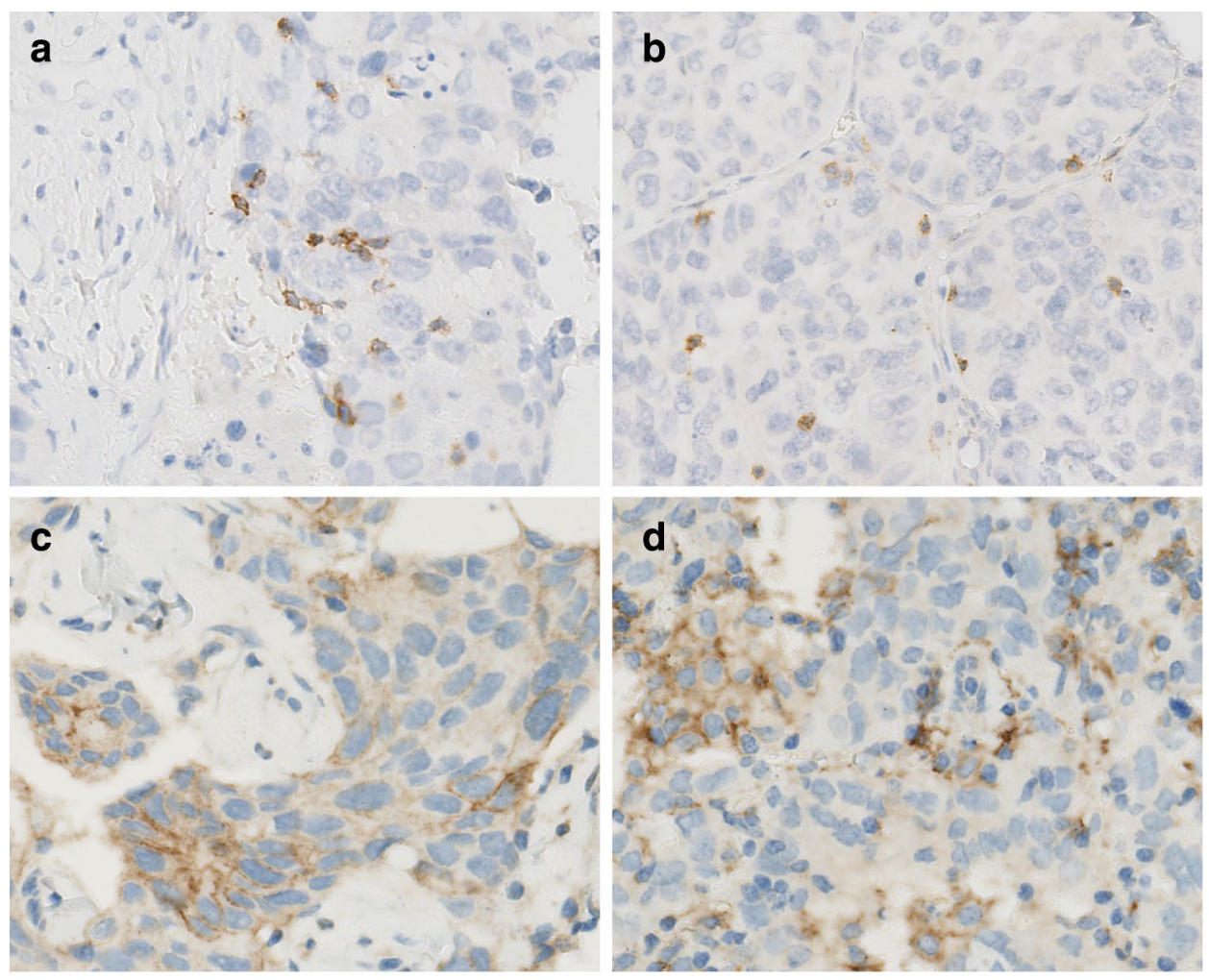

Table 4 PD-1 and PD-L1 expression in primary tumors and nonmatched distant metastases

\begin{tabular}{llll}
\hline Characteristics & Primary breast tumor & Metastasis & p-value* \\
\hline $\begin{array}{l}\text { PD-1 [no. (\%)] } \\
\text { Negative }\end{array}$ & $46(43.8)$ & $46(52.9)$ & 0.211 \\
$\begin{array}{l}\text { Positive } \\
\text { PD-L1 tumor }\end{array}$ & $59(56.2)$ & $41(47.1)$ & \\
cells [no. (\%)] & & & \\
Negative & $57(76.0)$ & $57(82.6)$ & 0.329 \\
Positive & $18(24.0)$ & $12(17.4)$ & \\
PD-L1 immune & & & \\
cells [no. (\%)] & & $38(55.1)$ & 0.785 \\
Negative & $43(57.3)$ & $31(44.9)$ & \\
Positive & $32(42.7)$ & & \\
\hline
\end{tabular}

\section{PD-1 and PD-L1 expression for various anatomic sites}

When comparing PD-1 and PD-L1 expression between various anatomic sites, least expression for PD-1 was seen in bone and gynecological tract metastases and most frequent PD-1 expression was observed in lung/pleural metastases. For tumor PD-L1 no clear differences were seen, while for immune PD-L1 all three bone metastases did not show any expression, and most expression was observed in lung/ pleural metastases, and also both gastrointestinal metastases were positive (Table 7).

*Pearson Chi square test 
Table 5 PD-1 and PD-L1 expression in primary breast tumors compared to their matched distant metastases

\begin{tabular}{|c|c|c|c|c|c|c|c|c|c|}
\hline \multicolumn{10}{|c|}{ Distant metastases } \\
\hline \multirow[t]{2}{*}{ Primary tumor } & \multicolumn{3}{|l|}{ PD-1 } & \multicolumn{3}{|c|}{ Tumor PD-L1 } & \multicolumn{3}{|c|}{ Immune PD-L1 } \\
\hline & Negative & Positive & p-value* & Negative & Positive & p-value* & Negative & Positive & p-value* \\
\hline Negative & $18(22.0 \%)$ & $17(20.7 \%)$ & 0.349 & $31(63.3 \%)$ & $3(6.1 \%)$ & 0.057 & $15(30.6 \%)$ & $10(20.4 \%)$ & 1.000 \\
\hline Positive & $24(29.3 \%)$ & $23(28.0 \%)$ & & $11(22.4 \%)$ & $4(8.2 \%)$ & & $10(20.4 \%)$ & $14(28.6 \%)$ & \\
\hline
\end{tabular}

*McNemar test

Table 6 Receptor conversion of primary breast tumors $(\mathrm{P})$ compared to their matched distant metastases (M)

\begin{tabular}{|c|c|c|c|c|c|c|c|}
\hline & $\begin{array}{l}\mathrm{PD}-1 \\
\mathrm{P}=\mathrm{M}\end{array}$ & $\begin{array}{l}\mathrm{PD}-1 \\
\mathrm{P} \neq \mathrm{M}\end{array}$ & p-value* & & $\begin{array}{l}P D-1 \\
P=M\end{array}$ & $\begin{array}{l}P D-1 \\
P \neq M\end{array}$ & p-value* \\
\hline $\begin{array}{l}\text { Tumor PD-L1 } \\
\mathrm{P}=\mathrm{M}\end{array}$ & $17(37.0 \%)$ & $16(34.8 \%)$ & 0.539 & $\begin{array}{l}\text { Immune PD-L1 } \\
\mathrm{P}=\mathrm{M}\end{array}$ & $17(37.0 \%)$ & $10(21.7 \%)$ & 0.162 \\
\hline $\begin{array}{l}\text { Tumor PD-L1 } \\
\mathrm{P} \neq \mathrm{M}\end{array}$ & $8(17.4 \%)$ & $5(10.9 \%)$ & & $\begin{array}{l}\text { Immune PD-L1 } \\
\mathrm{P} \neq \mathrm{M}\end{array}$ & $8(17.4 \%)$ & $11(23.9 \%)$ & \\
\hline
\end{tabular}

*Pearson Chi square test

Table 7 PD-1 and PD-L1 expression in breast cancer metastases at different anatomical sites

\begin{tabular}{|c|c|c|c|c|c|c|}
\hline \multirow[t]{2}{*}{ Location metastasis } & \multicolumn{2}{|l|}{ PD-1 } & \multicolumn{2}{|l|}{ Tumor PD-L1 } & \multicolumn{2}{|l|}{ Immune PD-L1 } \\
\hline & Negative no. (\%) & Positive No. (\%) & Negative no. (\%) & Positive no. (\%) & Negative no. (\%) & Positive no. (\%) \\
\hline Bone (marrow) & $4(100)$ & $0(0.0)$ & $3(100)$ & $0(0.0)$ & $3(100)$ & $0(0.0)$ \\
\hline Brain & $17(56.7)$ & $13(43.3)$ & $24(88.9)$ & $3(11.1)$ & $16(59.3)$ & $11(40.7)$ \\
\hline Gastrointestinal $^{\mathrm{a}}$ & $1(33.3)$ & $2(66.7)$ & $1(50.0)$ & $1(50.0)$ & $0(0.0)$ & $2(100)$ \\
\hline Gynecological & $4(80.0)$ & $1(20.0)$ & $3(100)$ & $0(0.0)$ & $2(66.7)$ & $1(33.3)$ \\
\hline Lung/pleural & $5(31.3)$ & $11(68.8)$ & $8(61.5)$ & $5(38.5)$ & $3(23.1)$ & $10(76.9)$ \\
\hline Skin/subcutis & $13(52.0)$ & $12(48.0)$ & $13(81.3)$ & $3(18.8)$ & $10(62.5)$ & $6(37.5)$ \\
\hline$p$-value* & 0.120 & & 0.238 & & 0.039 & \\
\hline
\end{tabular}

Bold indicates $p<0.05$

The italic means that $\mathrm{p}$-value does not belong to the metastasis location

*Pearson Chi square test

${ }^{a}$ For the gastrointestinal subgroup, also liver metastases were included $(\mathrm{n}=2)$

\section{Associations between PD-1 and PD-L1 expression and clinicopathological variables}

In both primary breast tumors and distant metastases, we did not observe correlations between PD-1 or tumor PD-L1 and hormone receptor status. Immune PD-L1 expression was associated with ER negativity in primary tumors, but not in metastases (Table 8). Further, in primary tumors, there was a correlation between PD-1 and a higher tumor grade $(\mathrm{p}<0.001)$, tumor PD-L1 and triple negative breast cancer $(p=0.003)$, and a tendency to an association was observed for immune PD-L1 and grade 3 tumors $(\mathrm{p}=0.078)$.

\section{Survival analysis}

Sufficient overall survival (OS) data were available for 66 patients. Of those, PD-1 was available for 65 patients, and PD-L1 expression was known for 49 patients. The median follow-up time was 5.1 years (range 1.3-25.9 years). No prognostic values were observed for PD-1 expression (HR 1.150, CI 0.667-1.983, $\mathrm{p}=0.614)$, PD-L1 tumor cell expression (HR $0.730, \mathrm{CI} 0.321-1.657, \mathrm{p}=0.449)$, and PD-L1 immune cell expression (HR 0.930, CI 0.510-1.695, $\mathrm{p}=0.812$ ) in distant metastases [Fig. 2]. Also in multivariate analysis, PD-1 and PD-L1 in metastases did not show prognostic values for OS.

In subgroup analysis, we did not see significant differences in overall survival for PD-1 or tumor PD-L1 expression "conversion" (positive to negative, or negative to positive) 
Table 8 Association of PD-1 and PD-L1 with selected clinicopathological variables in primary breast tumors and their matched distant metastases

\begin{tabular}{|c|c|c|c|c|c|c|c|c|}
\hline PD-1 & & P-value* & Tumor PD-L1 & & P-value* & Immune PD-I & & P-value* \\
\hline $\begin{array}{l}\text { Negative no. } \\
(\%)\end{array}$ & $\begin{array}{l}\text { Positive no. } \\
(\%)\end{array}$ & & $\begin{array}{l}\text { Negative no. } \\
(\%)\end{array}$ & $\begin{array}{l}\text { Positive no. } \\
(\%)\end{array}$ & & $\begin{array}{l}\text { Negative no. } \\
(\%)\end{array}$ & $\begin{array}{l}\text { Positive no. } \\
(\%)\end{array}$ & \\
\hline
\end{tabular}

Primary tumor

ER

\begin{tabular}{|c|c|c|c|c|c|c|c|c|c|}
\hline Negative & $14(37.8)$ & $23(62.2)$ & 0.361 & $47(72.3)$ & 18 (27.7) & 0.101 & $29(44.6)$ & $36(55.4)$ & 0.017 \\
\hline Positive & $22(47.8)$ & $24(52.2)$ & & $62(83.8)$ & $12(16.2)$ & & 48 (64.9) & $26(35.1)$ & \\
\hline \multicolumn{10}{|l|}{ PR } \\
\hline Negative & $15(34.1)$ & $29(65.9)$ & 0.091 & $60(74.1)$ & $21(25.9)$ & 0.170 & $43(53.1)$ & $38(46.9)$ & 0.639 \\
\hline Positive & $20(52.6)$ & $18(47.4)$ & & 47 (83.9) & $9(16.1)$ & & $32(57.1)$ & $24(42.9)$ & \\
\hline \multicolumn{10}{|l|}{ HER2 } \\
\hline Negative & $28(42.4)$ & $38(57.6)$ & 0.765 & $83(76.1)$ & $26(23.9)$ & 0.129 & $59(54.1)$ & $50(45.9)$ & 0.532 \\
\hline Positive & $7(46.7)$ & $8(53.3)$ & & $25(89.3)$ & $3(10.7)$ & & $17(60.7)$ & $11(39.3)$ & \\
\hline \multicolumn{10}{|l|}{ Metastasis } \\
\hline \multicolumn{10}{|l|}{ ER } \\
\hline Negative & $17(43.6)$ & $22(56.4)$ & 0.126 & $24(77.4)$ & 7 (22.6) & 0.447 & $14(45.2)$ & $17(54 . .8)$ & 0.216 \\
\hline Positive & $26(60.5)$ & $17(39.5)$ & & $28(84.8)$ & $5(15.2)$ & & $20(60.6)$ & $13(39.4)$ & \\
\hline \multicolumn{10}{|l|}{ PR } \\
\hline Negative & $26(51.0)$ & $25(49.0)$ & 0.620 & 35 (81.4) & 8 (18.6) & 1.000 & $24(55.8)$ & $19(44.2)$ & 0.424 \\
\hline Positive & $17(56.7)$ & $13(43.3)$ & & $16(80.0)$ & $4(20.0)$ & & $9(45.0)$ & $11(55.0)$ & \\
\hline \multicolumn{10}{|l|}{ HER2 } \\
\hline Negative & $34(52.3)$ & $31(47.7)$ & 0.963 & $43(82.7)$ & $9(17.3)$ & 0.682 & 27 (51.9) & $25(48.1)$ & 0.688 \\
\hline Positive & $9(52.9)$ & $8(47.1)$ & & $9(75.0)$ & $3(25.0)$ & & $7(58.3)$ & $5(41.7)$ & \\
\hline
\end{tabular}

Bold indicates $p<0.05$

*Pearson Chi square test
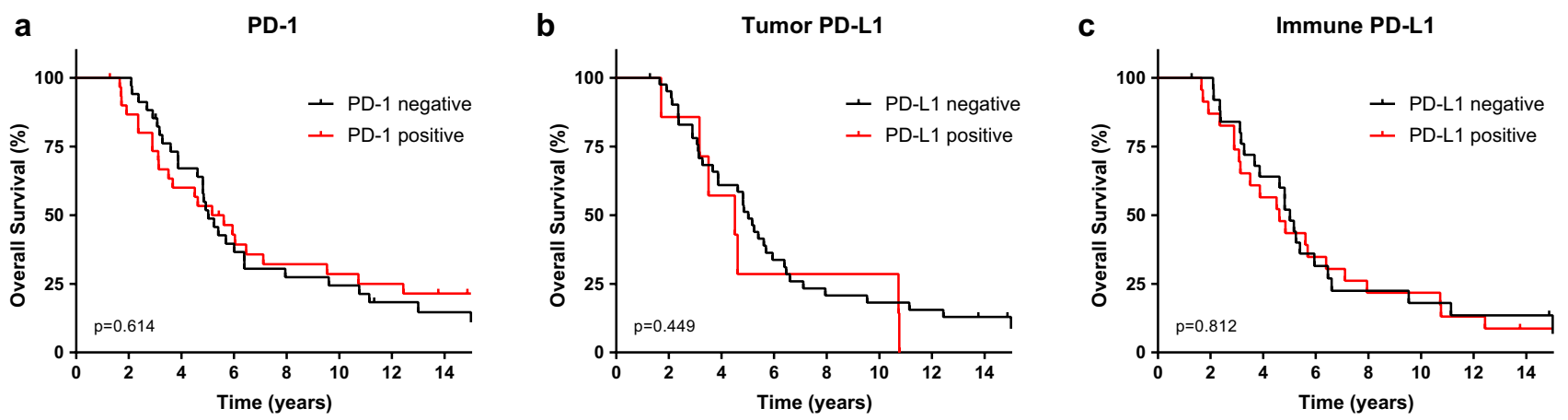

Fig. 2 Kaplan-Meier curves for Overall Survival (OS) for PD-1 (a), PD-L1 on tumor cells (b), and PD-L1 on immune cells (c) expression in distant metastases

versus "similarity" (positive to positive, or negative to negative) between primary breast tumor and metastasis $(\mathrm{p}=0.848$ and $\mathrm{p}=0.449$ ). For immune PD-L1, a significantly better OS was observed for patients with PD-L1 negative primary breast tumors that developed PD-L1 positive distant metastases $(p=0.044)$. The hazard ratio for this group compared to the other three groups combined was 3.013 (CI 1.201-7.561, $\mathrm{p}=0.019$ ).

\section{Discussion}

The aim of our study was to compare expression levels of PD- 1 on immune cells, and PD-L1 on tumor cells and immune cells, in primary tumors and their matched distant metastases in a large group of breast cancer patients, and to evaluate prognostic values. 
We observed that PD-1 and PD-L1 tumor and immune expression were concordant in the distant metastasis and its primary breast tumor in about half to two-third of the patients. In the other part of the patients, PD-1 or PD-L1 negative tumors had developed PD-1 or PD-L1 positive metastases or vice versa. Cimino-Mathews et al. also observed such discordance, but only in two patients and solely for the PD-L1 positive primary tumors [23]. Other studies in breast cancer observed concordance in expression of PD-L1 for primary breast tumors and matched (nodal) metastases in $94 \%$ and $100 \%$ [22, 29], which might be due to the relatively small size of their cohorts (15 and 17 matched cases, respectively). No previous studies that have been executed, describe PD-1 expression in breast cancer compared to matched distant metastases. Schneider et al. observed high concordance of PD-1 expression in primary head and neck squamous cell carcinoma and matched lymph node metastases $(90 \%)$, while a slightly lower concordance percentage was found for PD-L1 (70\%) [30]. Additionally, we observed that if PD-1 or PD-L1 was discordant in the distant metastasis compared to the primary tumor, this was not necessarily the case for the other protein. As far as we know we are the first to observe this inconsistency. The underlying biology for this discrepancy is yet unknown.

Possible explanations for discrepancies between primary tumors and their matched metastases may be genomic evolution during tumor progression [31], or clonal selection during metastatic process [18]. Otherwise adjuvant (chemo) therapy might influence the tumor microenvironment in breast cancer, and within that process, PD-1 or PD-L1 expression might change. This has been observed before by Yoon et al. where PD-L1 expression levels changed in 4/14 patients treated with neoadjuvant anthracycline- and/ or taxane-based chemotherapy [32]. Additionally, Pelekanou et al. compared both TILs and PD-L1 expression in tumors of 58 patients pre- and post-neoadjuvant chemotherapy, and observed an increase of TILs, but a decrease of PD-L1 expression in the post-neoadjuvant therapy tumors [33]. According to these results it may be interesting for future studies to look into more detail into the role of adjuvant therapy in relation to PD-1 and PD-L1 expression conversion in distant metastases.

As we compared PD-1 and PD-L1 expression in different distant anatomic sites, we observed some expression differences between those anatomic sites, in particular for PD-1 and immune PD-L1, although numbers were too small for proper statistical analysis. For example, gynaecological metastases less frequently showed PD-1 expression, while all four bone metastases were negative for PD-1. Previous studies, both in breast cancer as in other cancer types, evaluated PD-L1 expression in lymph node or clustered distant metastases [21, 22, 30, 34], which impedes comparison of our observations. However, Ogiya et al. studied PD-L1 expression in brain metastases only, and observed comparable results with regard to PD-L1 expression frequency as we $\operatorname{did}(45 \%$ vs. $41 \%)$ [24].

Furthermore, in subgroup analysis, we found a correlation between PD-1 positivity and higher tumor grade in primary breast tumors, which is in line with literature $[8,14,16]$. We did not observe other associations between PD-1 expression and selected clinicopathological variables, both in primary breast tumors as in metastases. Concerning PD-L1 in primary breast tumors, we observed associations of immune PD-L1 with ER negativity and a tendency of a correlation with grade 3 tumors, and for tumor PD-L1 an association with triple negative breast cancer. These findings are in line with the literature, since PD-L1 positivity is often associated with worse clinicopathological characteristics like larger tumor size, higher tumor grade and ER and PR negativity [7, 9-11, 13, 15]. The relatively small size of our cohort for subgroup analyses might explain we only observed these associations in primary tumors and not in metastases.

In survival analysis we did not observe significant differences concerning OS for PD-1 and PD-L1 on both tumor and immune cells. As far as we know, no other study has assessed prognostic value of PD-1 and PD-L1 expression in metastases. In primary breast tumors, the prognostic value of PD-1 and PD-L1 is equivocal, since previous studies described different outcomes for both PD-1 $[14,16]$, as PD-L1 $[6,7,10,11,15]$. Furthermore, a significantly better OS for patients with PD-L1 negative tumors that developed PD-L1 positive metastases was observed in the present study. However, subgroups were small, so we do not want to speculate on the biological background of this observation. Further evaluation in bigger groups could be interesting.

Comparison of PD-1 and PD-L1 expression between different studies remains difficult due to use of different antibodies, scoring methods and use of different cut-off values. For example, cut-off values varying from 1 to $50 \%$ were used, leading to different percentages of positive tumors [17]. Additionally, PD-L1 is expressed on both tumor cells and immune cells. Whereas most studies evaluate PD-L1 on tumor cells, recent studies also considered PD-L1 expression on immune cells $[10,17]$, like we did. Therefore, further standardization of (scoring) methods for the use of PD-1 and PD-L1 seems to be important.

Since PD-1 and PD-L1 expression are thought to be heterogeneous, it might be a limitation that we used TMAs to evaluate expression instead of full tissue slides. We therefore used three cores per tissue block that represented different tumor areas. Additionally, we verified the use of TMAs by comparing these PD-L1 scores of ten patients with PD-L1 scores on whole slides. In half of the patients complete agreement was noticed, while the other half only showed minimal differences. The strength of our study lays in the 
relatively large group of primary tumors and matched distant metastases of breast cancer patients.

To conclude, PD-1 and PD-L1 (on tumor and immune cells) positivity is concordant between primary tumors and distant metastases in only half to two-third of the breast cancer patients. In the other part of the patients PD-1 or PD-L1 negative tumors developed PD-1 or PD-L1 positive metastases or vice versa. Furthermore, patients with immune PD-L1 negative breast tumors that develop PD-L1 positive distant metastases seem to have the best overall survival. This illustrates the need of reassessing PD- 1 and tumor and immune PD-L1 expression in biopsies of distant metastases to optimize the usefulness of these biomarkers.

Acknowledgements We would like to thank the tissue facility of the UMCU for their help with the immunohistochemical stainings.

Funding Quirine F. Manson is financially supported by the Dutch Cancer Society, Grant No. KUN2014-7032.

\section{Compliance with ethical standards}

Conflict of interest The authors declare that they have no conflict of interest.

Open Access This article is distributed under the terms of the Creative Commons Attribution 4.0 International License (http://creativeco mmons.org/licenses/by/4.0/), which permits unrestricted use, distribution, and reproduction in any medium, provided you give appropriate credit to the original author(s) and the source, provide a link to the Creative Commons license, and indicate if changes were made.

\section{References}

1. Gatalica Z et al (2014) Programmed cell death 1 (PD-1) and its ligand (PD-L1) in common cancers and their correlation with molecular cancer type. Cancer Epidemiol Biomark Prev 23(12):2965-2970

2. Konishi J et al (2004) B7-H1 expression on non-small cell lung cancer cells and its relationship with tumor-infiltrating lymphocytes and their PD-1 expression. Clin Cancer Res 10(15):5094-5100

3. Hino R et al (2010) Tumor cell expression of programmed cell death-1 ligand 1 is a prognostic factor for malignant melanoma. Cancer 116(7):1757-1766

4. Hamanishi $\mathbf{J}$ et al (2007) Programmed cell death 1 ligand 1 and tumor-infiltrating $\mathrm{CD} 8+\mathrm{T}$ lymphocytes are prognostic factors of human ovarian cancer. Proc Natl Acad Sci USA 104(9):3360-3365

5. Wang X et al (2016) PD-L1 expression in human cancers and its association with clinical outcomes. OncoTargets Ther 9:5023-5039

6. Ali HR et al (2015) PD-L1 protein expression in breast cancer is rare, enriched in basal-like tumours and associated with infiltrating lymphocytes. Ann Oncol 26(7):1488-1493

7. Baptista MZ et al (2016) Prognostic significance of PD-L1 and PD-L2 in breast cancer. Hum Pathol 47(1):78-84
8. Ghebeh H et al (2008) FOXP3+ Tregs and B7-H1+/PD-1+ T lymphocytes co-infiltrate the tumor tissues of high-risk breast cancer patients: implication for immunotherapy. BMC Cancer 8:57

9. Ghebeh $\mathrm{H}$ et al (2006) The B7-H1 (PD-L1) T lymphocyte-inhibitory molecule is expressed in breast cancer patients with infiltrating ductal carcinoma: correlation with important high-risk prognostic factors. Neoplasia 8(3):190-198

10. Guo L et al (2016) PD-L1 expression and CD274 gene alteration in triple-negative breast cancer: implication for prognostic biomarker. Springerplus 5(1):805

11. Li Z et al (2016) PD-L1 expression is associated with tumor FOXP3(+) regulatory T-cell infiltration of breast cancer and poor prognosis of patient. J Cancer 7(7):784-793

12. Mittendorf EA et al (2014) PD-L1 expression in triple-negative breast cancer. Cancer Immunol Res 2(4):361-370

13. Muenst $\mathrm{S}$ et al (2014) Expression of programmed death ligand 1 (PD-L1) is associated with poor prognosis in human breast cancer. Breast Cancer Res Treat 146(1):15-24

14. Muenst $\mathrm{S}$ et al (2013) The presence of programmed death 1 (PD1)-positive tumor-infiltrating lymphocytes is associated with poor prognosis in human breast cancer. Breast Cancer Res Treat 139(3):667-676

15. Qin T et al (2015) High PD-L1 expression was associated with poor prognosis in 870 Chinese patients with breast cancer. Oncotarget 6(32):33972-33981

16. Sun $\mathrm{S}$ et al (2014) PD-1(+) immune cell infiltration inversely correlates with survival of operable breast cancer patients. Cancer Immunol Immunother 63(4):395-406

17. Sun WY, Lee YK, Koo JS (2016) Expression of PD-L1 in triplenegative breast cancer based on different immunohistochemical antibodies. J Transl Med 14(1):173

18. Hoefnagel LD et al (2013) Discordance in ERalpha, PR and HER2 receptor status across different distant breast cancer metastases within the same patient. Ann Oncol 24(12):3017-3023

19. Vermeulen JF et al (2012) Immunophenotyping invasive breast cancer: paving the road for molecular imaging. BMC Cancer $12: 240$

20. Hoefnagel LD et al (2012) Prognostic value of estrogen receptor alpha and progesterone receptor conversion in distant breast cancer metastases. Cancer 118(20):4929-4935

21. Dill EA et al (2017) PD-L1 expression and intratumoral heterogeneity across breast cancer subtypes and stages: an assessment of 245 primary and 40 metastatic tumors. Am J Surg Pathol 41(3):334-342

22. Tawfik O et al (2018) Clinicopathological correlation of PD-L1 expression in primary and metastatic breast Cancer and infiltrating immune cells. Hum Pathol. https://doi.org/10.1016/j.humpa th. 2018.06 .008

23. Cimino-Mathews A et al (2016) PD-L1 (B7-H1) expression and the immune tumor microenvironment in primary and metastatic breast carcinomas. Hum Pathol 47(1):52-63

24. Ogiya R et al (2017) Comparison of immune microenvironments between primary tumors and brain metastases in patients with breast cancer. Oncotarget 8(61):103671-103681

25. Heerma van Voss MR et al (2017) The prognostic effect of DDX3 upregulation in distant breast cancer metastases. Clin Exp Metastasis 34(1):85-92

26. Jiwa LS et al (2014) Upregulation of Claudin-4, CAIX and GLUT-1 in distant breast cancer metastases. BMC Cancer 14:864

27. Elston CW, Ellis IO (2002) Pathological prognostic factors in breast cancer. I. The value of histological grade in breast cancer: experience from a large study with long-term follow-up. Histopathology 41(3A):154-161

28. van Diest PJ (2002) No consent should be needed for using leftover body material for scientific purposes. For BMJ 325(7365):648-651 
29. Dill EA et al (2018) IDO expression in breast cancer: an assessment of 281 primary and metastatic cases with comparison to PD-L1. Mod Pathol 31:1513-1522

30. Schneider S et al (2018) PD-1 and PD-L1 expression in HNSCC primary cancer and related lymph node metastasis-impact on clinical outcome. Histopathology. https://doi.org/10.1111/ his. 13646

31. Polyak K (2007) Breast cancer: origins and evolution. J Clin Invest 117(11):3155-3163
32. Yoon HK et al (2018) Effect of anthracycline and taxane on the expression of programmed cell death ligand-1 and galectin-9 in triple-negative breast cancer. Pathol Res Pract 214(10):1626-1631

33. Pelekanou V et al (2017) Effect of neoadjuvant chemotherapy on tumor-infiltrating lymphocytes and PD-L1 expression in breast cancer and its clinical significance. Breast Cancer Res 19(1):91

34. Wang HB et al (2017) Rise of PD-L1 expression during metastasis of colorectal cancer: implications for immunotherapy. J Dig Dis 18(10):574-581 